Bull. Korean Math. Soc. 49 (2012), No. 2, pp. 435-443

http://dx.doi.org/10.4134/BKMS.2012.49.2.435

\title{
T-STRUCTURE AND THE YAMABE INVARIANT
}

\author{
Chanyoung Sung
}

\begin{abstract}
The Yamabe invariant is a topological invariant of a smooth closed manifold, which contains information about possible scalar curvature on it. It is well-known that a product manifold $T^{m} \times B$ where $T^{m}$ is the $m$-dimensional torus, and $B$ is a closed spin manifold with nonzero $\hat{A}$-genus has zero Yamabe invariant.

We generalize this to various $T$-structured manifolds, for example $T^{m}$ bundles over such $B$ whose transition functions take values in $\operatorname{Sp}(m, \mathbb{Z})$ (or $S p(m-1, \mathbb{Z}) \oplus\{ \pm 1\}$ for odd $m$ ).
\end{abstract}

\section{Introduction to Yamabe invariant}

The Yamabe invariant is an invariant of a smooth closed manifold depending on its smooth topology.

Let $M$ be a smooth closed manifold of dimension $n$. Given a smooth Riemannian metric $g$ on it, the conformal class $[g]$ is defined as

$$
[g]=\left\{\varphi g \mid \varphi: M \rightarrow \mathbb{R}^{+} \text {is smooth }\right\} .
$$

The famous Yamabe problem ([13]) states that there exists a metric $\tilde{g}$ in $[g]$ which attains the minimum

$$
\inf _{\tilde{g} \in[g]} \frac{\int_{M} s_{\tilde{g}} d V_{\tilde{g}}}{\left(\int_{M} d V_{\tilde{g}}\right)^{\frac{n-2}{n}}},
$$

where $s_{\tilde{g}}$ and $d V_{\tilde{g}}$ respectively denote the scalar curvature and the volume element of $\tilde{g}$.

It turns out that when $n \geq 3$, a unit-volume minimizer $\tilde{g}$ in $[g]$ has constant scalar curvature, which is equal to the above minimum value called the Yamabe constant of $[g]$ and denoted by $Y(M,[g])$.

It is known that the Yamabe constant of any $n$-manifold is bounded above by $Y\left(S^{n},\left[g_{0}\right]\right)$ where $\left[g_{0}\right]$ denotes a standard round metric. Thus following a

Received December 23, 2010

2010 Mathematics Subject Classification. 53C20, 55R10.

Key words and phrases. Yamabe invariant, T-structure, torus bundle.

This work was supported by the National Research Foundation of Korea(NRF) grant funded by the Korea government(MEST) (No. 2011-0002791, 2011-0001565). 
min-max procedure we define the Yamabe invariant

$$
Y(M):=\sup _{[g]} Y(M,[g])
$$

of $M$.

The following facts are noteworthy.

- $Y(M)>0$ if and only if $M$ admits a metric of positive scalar curvature.

- If $M$ is simply-connected and $\operatorname{dim} M \geq 5$, then $Y(M) \geq 0$. With the further assumption that $M$ is spin, $Y(M)>0$ if and only if the $\alpha$-genus of $M$ is 0 .

- For $r \in\left[\frac{n}{2}, \infty\right]$,

$$
|Y(M,[g])|=\inf _{\tilde{g} \in[g]}\left(\int_{M}\left|s_{\tilde{g}}\right|^{r} d \mu_{\tilde{g}}\right)^{\frac{1}{r}}\left(\operatorname{Vol}_{\tilde{g}}\right)^{\frac{2}{n}-\frac{1}{r}},
$$

where the infimum is attained only by the Yamabe minimizers.

- When $Y(M,[g]) \leq 0$,

$$
Y(M,[g])=-\inf _{\tilde{g} \in[g]}\left(\int_{M}\left|s_{\tilde{g}}^{-}\right|^{r} d \mu_{\tilde{g}}\right)^{\frac{1}{r}}\left(\operatorname{Vol}_{\tilde{g}}\right)^{\frac{2}{n}-\frac{1}{r}},
$$

where $s_{g}^{-}$is defined as $\min \left\{s_{g}, 0\right\}$.

Therefore when $Y(M) \leq 0$,

$$
\begin{aligned}
Y(M) & =-\inf _{g}\left(\int_{M}\left|s_{g}\right|^{r} d \mu_{g}\right)^{\frac{1}{r}}\left(\operatorname{Vol}_{g}\right)^{\frac{2}{n}-\frac{1}{r}} \\
& =-\inf _{g}\left(\int_{M}\left|s_{g}^{-}\right|^{r} d \mu_{g}\right)^{\frac{1}{r}}\left(\operatorname{Vol}_{g}\right)^{\frac{2}{n}-\frac{1}{r}},
\end{aligned}
$$

so that $Y(M)$ measures how much negative scalar curvature is inevitable on $M$.

- As an application of the above formula, if $M$ has an $F$-structure which will be explained in a later section, $M$ admits a sequence of metrics with volume form converging to zero while the sectional curvature are bounded below, so that $Y(M) \geq 0$ (See [14]).

\section{Computation of Yamabe invariant}

We now discuss how to compute the Yamabe invariant. When $M$ is a closed oriented surface, by the Gauss-Bonnet theorem

$$
Y(M)=4 \pi \chi(M),
$$

where $\chi$ denotes the Euler characteristic.

When $M$ is a closed oriented 3-manifold, the Ricci flow gave many answers by the proof of geometrization theorem due to G. Perelman (See [1]). For example, $Y(M)>0$ if and only if $M$ is a connected sum of $S^{1} \times S^{2}$ 's and finite quotients of $S^{3}$, and $Y\left(\mathbb{H}^{3} / \Gamma\right)$ is realized by the hyperbolic metric.

When $\operatorname{dim} M=4$, the Seiberg-Witten theory enables us to compute the Yamabe invariant of Kähler surfaces through the Weitzenböck formula. LeBrun 
$[10,11,12]$ has shown that if $M$ is a compact Kähler surface whose Kodaira dimension is not equal to $-\infty$, then

$$
Y(M)=-4 \sqrt{2} \pi \sqrt{2 \chi(\tilde{M})+3 \tau(\tilde{M})}
$$

where $\tau$ denotes the signature and $\tilde{M}$ is the minimal model of $M$, and for $\mathbb{C} P^{2}$,

$$
Y\left(\mathbb{C} P^{2}\right)=4 \sqrt{2} \pi \sqrt{2 \chi\left(\mathbb{C} P^{2}\right)+3 \tau\left(\mathbb{C} P^{2}\right)} .
$$

In higher dimensions, few examples have been computed so far, such as

$$
Y\left(S^{1} \times S^{n-1}\right)=Y\left(S^{n}\right)=n(n-1)\left(\operatorname{vol}\left(S^{n}(1)\right)\right)^{\frac{2}{n}},
$$

where $S^{n}(1)$ is the unit sphere in $\mathbb{R}^{n+1}$, and

$$
Y\left(T^{n}\right)=Y\left(T^{n} \times H\right)=Y\left(T^{n} \times B\right)=0,
$$

where $H$ is a closed Hadarmard-Cartan manifold, i.e., one with a metric of nonpositive sectional curvature, and $B$ is a closed spin manifold with nonzero $\hat{A}$ genus. These $T^{n}$-bundles have such property, because they admit a $T$-structure and never admit a metric of positive scalar curvature by Gromov-Lawson enlargeability method [5, 9].

We call a closed $n$-manifold $M$ enlargeable if the following holds: for any $\epsilon>0$ and any Riemannian metric $g$ on $M$, there exists a Riemannian spin covering manifold $\tilde{M}$ of $(M, g)$ and an $\epsilon$-contracting map $f: \tilde{M} \rightarrow S^{n}(1)$, which is constant outside a compact subset of $\tilde{M}$ and of nonzero $\hat{A}$-degree defined as $\hat{A}\left(f^{-1}\right.$ (any regular value of $\left.\left.f\right)\right)$.

Here a smooth map $F$ is called $\epsilon$-contracting if the norm of $D F$ is less than $\epsilon$. By using the Weitzenböck formula for an appropriate twisted Dirac operator, they showed that such manifolds never admit a metric of positive scalar curvature.

They also generalized this to so-called weakly-enlargeable manifolds, where " $\epsilon$-contracting" is replaced by " $\epsilon$-contracting on 2 -forms" meaning that the induced map of $D F$ on tangent bi-vectors, i.e., a section of $\Lambda^{2}(T M)$ has norm less than $\epsilon$.

$\hat{A}$-genus of a closed spin manifold $M$ is the integral over $M$ of

$$
\hat{A}(T M):=1-\frac{p_{1}}{24}+\frac{-4 p_{2}+7 p_{1}^{2}}{5760}+\cdots,
$$

where $p_{i} \in H^{4 i}(M, \mathbb{Z})$ is the $i$-th Pontryagin class of $T M$. An important fact is that a closed spin manifold with a metric of positive scalar curvature has zero $\hat{A}$-genus.

Then a natural question for us to explore is:

Question 2.1. Let $M$ be a $T^{m}$-bundle over a closed spin manifold $B$ with nonzero $\hat{A}$-genus. Is $Y(M)$ equal to zero? 


\section{T-structure}

An F-structure which was introduced by Cheeger and Gromov [3, 4] generalizes an effective $T^{m}$-action for $m \in \mathbb{N}$.

Definition 3.1. An $F$-structure on a smooth manifold is given by data $\left(U_{i}, \hat{U}_{i}\right.$, $\left.T^{k_{i}}\right)$ with the following conditions:

(1) $\left\{U_{i}\right\}$ is a locally finite open cover.

(2) Each $\pi_{i}: \hat{U}_{i} \mapsto U_{i}$ is a finite Galois covering with covering group $\Gamma_{i}$.

(3) Each torus $T^{k_{i}}$ of dimension $k_{i}$ acts effectively on $\hat{U}_{i}$ in a $\Gamma_{i}$-equivariant way, i.e., $\Gamma_{i}$ also acts on $T^{k_{i}}$ as an automorphism so that

$$
\gamma(g x)=\gamma(g) \gamma(x)
$$

for any $\gamma \in \Gamma_{i}, g \in T^{k_{i}}$, and $x \in \hat{U}_{i}$.

(4) If $U_{i} \cap U_{j} \neq \emptyset$, then there is a common covering of $\pi_{i}^{-1}\left(U_{i} \cap U_{j}\right)$ and $\pi_{j}^{-1}\left(U_{i} \cap U_{j}\right)$ such that it is invariant under the lifted actions of $T^{k_{i}}$ and $T^{k_{j}}$, and they commute.

As a special case, a $T$-structure is an $F$-structure in which all the coverings $\pi_{i}$ 's are trivial.

Typical examples of $T$-structure are torus bundles.

Theorem 3.2. Any $T^{m}$-bundle over a smooth manifold whose transition functions are $T^{m} \rtimes G L(m, \mathbb{Z})$-valued has a $T$-structure. In particular, any $S^{1}$ or $T^{2}$-bundle has a T-structure.

Proof. Here $T^{m}$ acts by translation, and hence the transition functions are affine maps at each fiber direction. Obviously the local $T^{m}$ actions along the fiber are commutative on the intersections to give a global $T$-structure.

The second statement follows from the well-known fact that the diffeomorphism group of $T^{m}$ for $m=1,2$ is homotopically equivalent to $T^{m} \rtimes$ $G L(m, \mathbb{Z})$. Thus we may assume that the transition functions are $T^{m} \rtimes$ $G L(m, \mathbb{Z})$-valued.

Other typical examples are manifolds with a nontrivial smooth $S^{1}$ action. Such examples we will use are projective spaces such as $\mathbb{R} P^{n}, \mathbb{C} P^{n}, \mathbb{H} P^{n}$, and $\mathrm{CaP}^{2}$ (For the case of the Cayley plane which actually has an $S^{3}$-action, see [2]). Or one can construct more examples by gluing $T$-structured manifolds. For example graph manifolds are obtained by gluing Seifert-fibred 3-manifolds along the toral boundaries, and also:

Theorem 3.3 (Paternain and Petean [14]). Suppose $X$ and $Y$ are $n$-manifolds with $n>2$, which admit a T-structure. Then $X \# Y$ also admits a T-structure.

\section{Main results}

Motivated by Gromov-Lawson enlargeability technique, we prove: 
Theorem 4.1. Let $B$ be a closed spin manifold of dimension $4 d$ with nonzero $\hat{A}$-genus, and $M$ be a $T^{m}$-bundle over $B$ whose transition functions take values in $S p(m, \mathbb{Z})($ or $S p(m-1, \mathbb{Z}) \oplus\{ \pm 1\}$ for odd $m)$. Then

$$
Y(M)=0 .
$$

Proof. By Theorem 3.2, $M$ has a $T$-structure so that $Y(M) \geq 0$.

We only have to show that $M$ never admits a metric of positive scalar curvature. To the contrary, suppose that it admits such a metric $h$, and we will derive a contradiction. The basic idea is to apply the Bochner-type method to a twisted $\operatorname{Spin}^{c}$ bundle on $M$ whose topological index is nonzero.

First, we consider the case when $m$ is even, say $2 k$. Let $\Lambda$ denote a lattice in $\mathbb{R}^{2 k}$ so that $T^{2 k}=\mathbb{R}^{2 k} / \Lambda$. Take an integer $n \gg 1$. There is an obvious covering map from $\mathbb{R}^{2 k} / n \Lambda$ onto $\mathbb{R}^{2 k} / \Lambda$ of degree $n^{2 k}$, and we claim that this covering map can be extended to all the fibers in $M$ to give a covering $p: M_{n} \rightarrow M$. The following lemma justifies this:

Lemma 4.2. The same transition functions as $\mathbb{R}^{2 k} / \Lambda$-bundle $M$ give $\mathbb{R}^{2 k} / n \Lambda$ bundle $M_{n}$ with the covering projection $p$.

Proof. For a transition map $g_{\alpha \beta} \in S p(2 k, \mathbb{Z})$ downstairs, the same transition map $g_{\alpha \beta}$ upstairs is the unique lifting map which satisfies $p \circ g_{\alpha \beta}=g_{\alpha \beta} \circ p$ and sends 0 to 0 .

It only needs to be proved that the transition maps satisfy the axioms for the bundle, in particular the axiom $g_{\beta \gamma} \circ g_{\alpha \beta}=g_{\alpha \gamma}$. This follows from the uniqueness of the lifting map sending 0 to 0 (In fact, this cocycle condition holds without modulo $\mathbb{Z}$ ).

We endow $M_{n}$ with a metric $h_{n}:=p^{*} h$.

Lemma 4.3. There exists a closed 2-form $\omega$ on $M_{n}$ such that $\omega^{k+1}=0$ and it restricts to a generator of $H^{2}\left(T^{2 k}, \mathbb{Z}\right)$ at each fiber $T^{2 k}$.

Proof. For each $U \times T^{2 k}$ where $U$ is an open ball in $B$, take $\omega$ to be a standard symplectic form of $T^{2 k}$ representing a generator of $H^{2}\left(T^{2 k}, \mathbb{Z}\right)$. Since $\omega$ is invariant under $S p(2 k, \mathbb{Z})$, it is globally defined on $M_{n}$ (Note that the transition functions are locally constant). Obviously $\omega^{k+1}=0$ at each point.

Let $E$ be the complex line bundle on $M_{n}$ whose first Chern class is [ $\left.\omega\right]$. Take a connection $A^{E}$ of $E$ whose curvature 2-form $R^{E}=d A^{E}$ is equal to $-2 \pi i \omega$.

We claim that

$$
\left|R^{E}\right|_{h_{n}} \rightarrow 0 \quad \text { as } n \rightarrow \infty .
$$

Lemma 4.4. $\left|R^{E}\right|_{h_{n}}=O\left(\frac{1}{n^{2}}\right)$.

Proof. Take a local coordinate $\left(x_{1}, \ldots, x_{4 d}\right) \times\left(y_{1}, \ldots, y_{2 k}\right)$ of $B \times T^{2 k}$ so that

$$
\omega=d y_{1} \wedge d y_{2}+\cdots+d y_{2 k-1} \wedge d y_{2 k} .
$$


We will show that $\left|d y_{\mu}\right|_{h_{n}}=O\left(\frac{1}{n}\right)$ for all $\mu$. First,

for all $i, j, \mu$, and $\nu$. Thus

$$
\begin{gathered}
h_{n}\left(\frac{\partial}{\partial x_{i}}, \frac{\partial}{\partial x_{j}}\right)=h\left(\frac{\partial}{\partial x_{i}}, \frac{\partial}{\partial x_{j}}\right), \\
h_{n}\left(\frac{\partial}{\partial x_{i}}, \frac{\partial}{\partial y_{\mu}}\right)=n h\left(\frac{\partial}{\partial x_{i}}, \frac{\partial}{\partial y_{\mu}}\right), \\
h_{n}\left(\frac{\partial}{\partial y_{\mu}}, \frac{\partial}{\partial y_{\nu}}\right)=n^{2} h\left(\frac{\partial}{\partial y_{\mu}}, \frac{\partial}{\partial y_{\nu}}\right)
\end{gathered}
$$

$$
h_{n}=\left(\begin{array}{cc}
O(1) & O(n) \\
O(n) & O\left(n^{2}\right)
\end{array}\right),
$$

where the block division is according to the division by $x$ and $y$ coordinates, and

$$
\begin{aligned}
\left(h_{n}\right)^{-1} & =\frac{1}{\operatorname{det}\left(h_{n}\right)} \operatorname{adj}\left(h_{n}\right) \\
& =\frac{1}{O\left(n^{4 k}\right)}\left(\begin{array}{cc}
O\left(n^{4 k}\right) & O\left(n^{4 k-1}\right) \\
O\left(n^{4 k-1}\right) & O\left(n^{4 k-2}\right)
\end{array}\right) \\
& =\left(\begin{array}{cc}
O(1) & O\left(\frac{1}{n}\right) \\
O\left(\frac{1}{n}\right) & O\left(\frac{1}{n^{2}}\right)
\end{array}\right),
\end{aligned}
$$

which means $\left|d x_{i}\right|_{h_{n}}=O(1)$ and $\left|d y_{\mu}\right|_{h_{n}}=O\left(\frac{1}{n}\right)$ for all $i$ and $\mu$, completing the proof.

In order to use the Bochner argument, we need to show that $M_{n}$ is $\operatorname{spin}^{c}$. Using the orthogonal decomposition by $h_{n}$,

$$
T M_{n}=V \oplus H=V \oplus \pi^{*}(T B),
$$

where $V$ and $H$ respectively denote the vertical and horizontal space, and $\pi: M_{n} \rightarrow B$ be the torus bundle projection. Obviously $H$ is spin, because $B$ is spin. Since $V$ is a symplectic $\mathbb{R}^{2 k}$-vector bundle, it admits a compatible almost-complex structure, and hence it can be viewed as a $\mathbb{C}^{k}$-vector bundle. Thus

$$
w_{2}\left(M_{n}\right)=w_{2}(V)+w_{2}(H) \equiv c_{1}(V) \bmod 2
$$

meaning that $M_{n}$ is $\operatorname{spin}^{c}$. Let $S$ be the associated vector bundle to the $\operatorname{Spin}^{c}$ bundle over $M_{n}$ obtained using $\sqrt{\operatorname{det}_{\mathbb{C}} V}$.

Consider a twisted $\operatorname{spin}^{c}$ Dirac operator $D^{E}$ on $S \otimes E$ where $E$ is equipped with a connection $A^{E}$. The Weitzenböck formula says that

$$
\left(D^{E}\right)^{2}=\nabla^{*} \nabla+\frac{1}{4} s_{h_{n}}+\mathfrak{R}^{E}
$$

Here $\mathfrak{R}^{E}(\sigma \otimes v)=\sum_{i<j}\left(e_{i} e_{j} \sigma\right) \otimes\left(R_{e_{i}, e_{j}}^{E} v\right)$ where $\left\{e_{i}\right\}$ is an orthonormal frame for $\left(M_{n}, h_{n}\right)$. Note that

$$
\left|\mathfrak{R}^{E}\right|_{h_{n}} \leq C\left|R^{E}\right|_{h_{n}},
$$


where $C$ is a positive constant depending on the dimension of $M$. By taking $n$ sufficiently large, we can ensure that $s_{h_{n}}>\left|\mathfrak{R}^{E}\right|_{h_{n}}$ everywhere, and hence $\operatorname{ker} D^{E}=0$. Thus the index of the operator

$$
D_{+}^{E}: \Gamma\left(S_{+} \otimes E\right) \rightarrow \Gamma\left(S_{-} \otimes E\right)
$$

is

$$
\left.\operatorname{dim} \operatorname{ker} D^{E}\right|_{S_{+} \otimes E}-\left.\operatorname{dim} \operatorname{ker} D^{E}\right|_{S_{-} \otimes E}=0,
$$

where $S_{ \pm}$respectively denotes the plus and negative spinor bundle.

On the other hand, we can also compute the index using the Atiyah-Singer index theorem [9]. Note that $V$ has locally constant transition functions, and hence can be given a flat connection. This implies that $\hat{A}(V)=1$ so that

$$
\begin{aligned}
\operatorname{index}\left(D_{+}^{E}\right) & =\left\{\operatorname{ch}(E) \cdot \hat{A}\left(T M_{n}\right)\right\}\left[M_{n}\right] \\
& =\left\{\left(1+[\omega]+\cdots+\frac{1}{k !}[\omega]^{k}\right) \cdot \hat{A}(V) \cdot \hat{A}\left(\pi^{*}(T B)\right)\right\}\left[M_{n}\right] \\
& =\left\{\left(1+[\omega]+\cdots+\frac{1}{k !}[\omega]^{k}\right) \cdot \pi^{*}(\hat{A}(B))\right\}\left[M_{n}\right] \\
& =\left\{\frac{1}{k !}[\omega]^{k} \cdot \pi^{*}\left(\hat{A}_{d}(B)\right)\right\}\left[M_{n}\right] \\
& =\int_{\pi^{-1}\left(P D\left[\hat{A}_{d}(B)\right]\right)} \frac{1}{k !}[\omega]^{k} \\
& =(\hat{A}(B)[B]) \int_{\pi^{-1}(p t)} \frac{1}{k !}[\omega]^{k} \\
& \neq 0,
\end{aligned}
$$

which yields a contradiction.

The odd $m$ case is reduced to the even case. If $m$ is odd, consider an $S^{1}$-bundle over $M$ with transition functions exactly equal to the transition functions $\{ \pm 1\}$ of the last $S^{1}$-factor of $T^{m}$ in $M$ over $B$. Then $M^{\prime}$ is a $T^{m+1}$ bundle over $B$ with transition functions taking values in $S p(m+1, \mathbb{Z})$. We put a locally product metric on $M^{\prime}$. Then it also has positive scalar curvature, yielding a contradiction from the above even case.

Theorem 4.5. Let $B$ be a closed spin manifold of dimension $4 d$ with nonzero $\hat{A}$-genus, and $M$ be an $S^{1}$ or $T^{2}$-bundle over $B$ whose transition functions take values in $G L(1, \mathbb{Z})$ or $G L(2, \mathbb{Z})$ respectively. Then

$$
Y(M)=0 \text {. }
$$

Proof. Again by Theorem 3.2, $M$ has a $T$-structure so that $Y(M) \geq 0$. It remains to show $M$ never admits a metric of positive scalar curvature, and let's assume it does.

First, the case of $S^{1}$ bundle can be reduced to the case of $T^{2}$ bundle by considering a Riemannian product $M \times S^{1}$ which also has positive scalar curvature. From now on, we consider the case of $T^{2}$ bundle. 
Secondly, we may also assume that $M$ is orientable, i.e., the transition functions for the torus bundle are orientation-preserving. Otherwise, we consider $\bar{M}$ from the lemma below, which also admits a metric of positive scalar curvature by lifting the metric of $M$.

Lemma 4.6. There exists a finite covering $\bar{M}$ of $M$ such that $\bar{M}$ is an orientable $T^{2}$-bundle over a closed spin manifold of nonzero $\hat{A}$-genus.

Proof. Let $\hat{B}$ be the universal cover of $B$, and $\hat{M}$ be the manifold obtained by lifting the torus bundle over $B$ to $\hat{B}$. Since $\hat{B}$ is simply-connected, $\hat{M}$ is orientable, and $\pi_{1}(B)$ acts on $\hat{M}$ to give $M=\hat{M} / \pi_{1}(B)$.

Let $G$ be a subset of $\pi_{1}(B)$, which consists of elements preserving orientation of the fiber torus. Then $G$ is a subgroup of index 2. Thus $\hat{M} / G$ is an orientable $T^{2}$-bundle over $\hat{B} / G$ which is a double cover of $B$ so that it is also spin with nonzero $\hat{A}$-genus.

Now if $M$ is orientable, its transition functions take values in $S L(2, \mathbb{Z})=$ $S p(2, \mathbb{Z})$ so that the previous theorem can be applied to derive a contradiction.

Remark 4.7. In fact, Theorem 4.1 holds for any $T^{m}$-bundle with $T^{m_{\rtimes}} G L(m, \mathbb{Z})$ valued transition functions, which has a finite covering diffeomorphic to $M$ as in Theorem 4.1.

Combining our results with the previous results in [16], we can compute more general $T$-structured manifolds:

Corollary 4.8. Let $M$ be a $T^{m}$-bundle in all the above so that $Y(M)=0$. If $\operatorname{dim} M=4 n$, then

$$
Y\left(M \sharp k \mathbb{H} P^{n_{\sharp}} l \overline{\mathbb{H} P^{n}}\right)=0,
$$

and if $\operatorname{dim} M=16$, then

$$
Y\left(M \sharp k \mathbb{H} P^{4} \sharp l \overline{\mathbb{H} P^{4}} \sharp k^{\prime} C a P^{2} \sharp l^{\prime} \overline{C a P^{2}}\right)=0,
$$

where $k, l, k^{\prime}$, and $l^{\prime}$ are nonnegative integers, and the overline denotes the reversed orientation.

In low dimensions such as 2 and 3, we understand all $T$-structured manifolds with zero Yamabe invariant. In dimension 4, we can compute the Yamabe invariant of some torus bundles by using the Seiberg-Witten theory.

Theorem 4.9. Let $B$ be a closed oriented manifold of dimension $\leq 3$, and $X$ be an $S^{1}$ or $T^{2}$-bundle over $B$. Suppose that $X \times T^{m}$ for $m=4-\operatorname{dim} X$ has a finite cover $M$ with $b_{2}^{+}(M)>1$ which is a $T^{2}$-bundle over an oriented surface whose transition functions take values in a discrete subset of $T^{2} \rtimes S L(2, \mathbb{Z})$. Then

$$
Y(X)=0
$$


Proof. It suffices to show that $M$ never admits a metric of positive scalar curvature.

Using the 2-form $\omega$ on $M$ which restricts to a standard symplectic form on each fiber, we have a symplectic form $\pi^{*} \sigma+\omega$ on $M$, where $\sigma$ is a symplectic form of $\tilde{B}$, and $\pi: M \rightarrow \tilde{B}$ is the $T^{2}$-bundle projection.

Then the Seiberg-Witten invariant of the canonical $\operatorname{Spin}^{c}$ structure of $M$ is \pm 1 so that it never admits a metric of positive scalar curvature.

\section{References}

[1] M. T. Anderson, Canonical metrics on 3-manifolds and 4-manifolds, Asian J. Math. 10 (2006), no. 1, 127-163.

[2] M. Atiyah and J. Berndt, Projective planes, Severi varieties and spheres, Surveys in differential geometry, Vol. VIII (Boston, MA, 2002), 1-27.

[3] J. Cheeger and M. Gromov, Collapsing Riemannian manifolds while keeping their curvature bounded I, J. Differential Geom. 23 (1986), no. 3, 309-346.

[4] _ Collapsing Riemannian manifolds while keeping their curvature bounded II, J. Differential Geom. 32 (1990), no. 1, 269-298.

[5] M. Gromov and H. B. Lawson, Positive scalar curvature and the Dirac operator on complete Riemannian manifolds, Inst. Hautes Études Sci. Publ. Math. No. 58 (1983), 83-196.

[6] M. J. Gursky and C. LeBrun, Yamabe invariants and spin ${ }^{c}$ structures, Geom. Funct. Anal. 8 (1998), no. 6, 965-977.

[7] M. Ishida and C. LeBrun, Curvature, connected sums, and Seiberg-Witten theory, Comm. Anal. Geom. 11 (2003), no. 5, 809-836.

[8] O. Kobayashi, Scalar curvature of a metric with unit volume, Math. Ann. 279 (1987), no. 2, 253-265.

[9] H. B. Lawson and M. L. Michelson, Spin Geometry, Priceton University Press, 1989.

[10] C. LeBrun, Four manifolds without Einstein metrics, Math. Res. Lett. 3 (1996), no. 2, $133-147$.

[11] _ Yamabe constants and the perturbed Seiberg-Witten equations, Comm. Anal. Geom. 5 (1997), no. 3, 535-553.

[12] _ Kodaira dimension and the Yamabe problem, Comm. Anal. Geom. 7 (1999), no. $1,133-156$

[13] J. Lee and T. Parker, The Yamabe problem, Bull. Amer. Math. Soc. (N.S.) 17 (1987), no. 1, 37-91.

[14] G. P. Paternain and J. Petean, Minimal entropy and collapsing with curvature bounded from below, Invent. Math. 151 (2003), no. 2, 415-450.

[15] J. Petean and G. Yun, Surgery and the Yamabe invariant, Geom. Funct. Anal. 9 (1999), no. 6, 1189-1199.

[16] C. Sung, Connected sums with $\mathbb{H} P^{n}$ or $C a P^{2}$ and the Yamabe invariant, arXiv:0710.2379

[17] _ Surgery and equivariant Yamabe invariant, Differential Geom. Appl. 24 (2006), no. $3,271-287$.

[18] _ Surgery, Yamabe invariant, and Seiberg-Witten theory, J. Geom. Phys. 59 (2009), no. 2, 246-255.

Department of Mathematics and Institute for Mathematical Sciences

KONKUK UNIVERSITY

SEOUL 143-701, KOREA

E-mail address: cysung@kias.re.kr 